\title{
New Measure for Shape Elongation
}

\author{
Miloš Stojmenović ${ }^{1}$ and Joviša Žuniće ${ }^{2, \star}$ \\ ${ }^{1}$ SITE, University of Ottawa, Ottawa, Ontario, Canada K1N 6N5 \\ mstoj075@site.uottawa.ca \\ ${ }^{2}$ Computer Science, Exeter University, Exeter EX4 4QF, U.K. \\ J.Zunic@ex.ac.uk
}

\begin{abstract}
Shape elongation is one of the basic shape descriptors that has a very clear intuitive meaning. That is reason for its applicability in many shape classification tasks. In this paper we define a new method for computing shape elongation for shapes with polygonal boundaries. The measure is the ratio of the maximal and minimal of the sums of squared lengths of the projections of all of the edges of the polygonal boundary onto a line which has a particular slope. We express the measure with a closed formula. This measure finds the elongation for shapes whose boundary is not extracted completely, which is impossible to achieve with existing area based measures.
\end{abstract}

Keywords: Shape, elongation, orientation, image processing, computer vision.

\section{Introduction}

This paper introduces a new shape elongation measure. Elongation has an intuitively clear meaning and is hence a very common shape descriptor. In literature, shape orientation and shape elongation are strongly connected, and usually considered together ([234] ). The standard measure of shape elongation is derived from the definition of shape orientation that is based on the axis of the least second moment of inertia. Precisely, the axis of the least second moment ([234]) is the line which minimises the integral of the squares of distances of the points (belonging to the shape) to the line. The integral is

$$
I(S, \varphi, \rho)=\iint_{S} r^{2}(x, y, \varphi, \rho) d x d y
$$

where $r(x, y, \varphi, \rho)$ is the perpendicular distance from the point $(x, y)$ to the line given in the form

$$
x \cdot \cos \varphi-y \cdot \sin \varphi=\rho .
$$

\footnotetext{
* The author is also with the Mathematical institute of Serbian Academy of Sciences and Arts, Belgrade.
} 
The angle $\varphi$ for which the above integral reaches a minimum defines the orientation of the shape $S$. This angle is easy to compute and it can be shown that such an angle $\varphi$ satisfies the following equation:

$$
\frac{\sin (2 \varphi)}{\cos (2 \varphi)}=\frac{2 \cdot \bar{m}_{1,1}(S)}{\bar{m}_{2,0}(S)-\bar{m}_{0,2}(S)}
$$

where $\bar{m}_{p, q}(S)$ are centralised moments of the shape $S$ defined as

$$
\bar{m}_{p, q}(S)=\iint_{S}\left(x-\frac{\iint_{S} x d x d y}{\iint_{S} d x d y}\right)^{p} \cdot\left(y-\frac{\iint_{S} y d x d y}{\iint_{S} d x d y}\right)^{q} d x d y
$$

The minimum and maximum of $I(S, \varphi, \rho)$ are also easy to compute. They are:

$$
\begin{aligned}
& \max _{\substack{\rho \geq 0 \\
\varphi \in[0,2 \pi]}}\{I(S, \varphi, \rho)\}= \\
& \frac{\bar{m}_{2,0}(S)+\bar{m}_{0,2}(S)+\sqrt{4 \cdot\left(\bar{m}_{1,1}(S)\right)^{2}+\left(\bar{m}_{2,0}(S)-\bar{m}_{0,2}(S)\right)^{2}}}{2}
\end{aligned}
$$

and

$$
\begin{aligned}
& \min _{\substack{\rho \geq 0 \\
\varphi \in[0,2 \pi]}}\{I(S, \varphi, \rho)\}= \\
& \frac{\bar{m}_{2,0}(S)+\bar{m}_{0,2}(S)-\sqrt{4 \cdot\left(\bar{m}_{1,1}(S)\right)^{2}+\left(\bar{m}_{2,0}(S)-\bar{m}_{0,2}(S)\right)^{2}}}{2} .
\end{aligned}
$$

Next, the ratio between $\max _{\varphi \in[0, \pi)} I(S, \varphi, \rho)$ and $\min _{\varphi \in[0, \pi)} I(S, \varphi, \rho)$

$$
\mathcal{E}_{s}(S)=\frac{\max \{I(S, \varphi, \rho) \mid \varphi \in[0,2 \cdot \pi], \quad \rho \geq 0\}}{\min \{I(S, \varphi, \rho) \mid \varphi \in[0,2 \cdot \pi], \quad \rho \geq 0\}}
$$

is the standard measure of elongation of the shape $S$. Some generalisation of the standard method for measuring shape elongation can be found in 8 . Let us mention that there are also some naive measures of elongation. For example, shape elongation can be measured as the ratio of the longer and shorter edges of the minimum area bounding rectangle for the measured shape. It is worth mentioning that such bounding rectangles are easy to compute ([1.5]).

The standard measure (4) of shape elongation is area based because all points belonging to the shape are involved in the computation (area moments are used). Our new shape elongation measure is boundary based, because only the boundary points are used in its computation. In this paper we will use the above given idea while considering a recently disclosed method [9] for computing shape orientation for deriving the new measure for shape elongation.

The restriction to polygonal shapes is not strictly enforced since real image processing applications deal with discrete data that are a result of a particular 
discretization process. In order to enhance the data manipulation, the boundaries of the original shapes are usually approximated with canonical arc sections (circular arcs, parabolic arcs, straight line segments, etc.). Approximating boundaries by straight line sections (i.e., polygonal approximation) is used most frequently and many algorithms for the polygonal shape approximation already exist - see [6].

The new elongation measure defined in this paper takes into account all the boundary points - not only those that belong to the convex hull or to bounding rectangles of the shape, for example.

\section{Boundary Based Shape Orientation}

As mentioned, we will derive a new shape elongation measure from a recent boundary based method for computing the orientation of polygonal shapes. We will first give a short sketch of the main result from 9. Let us start with the following definition from the same paper.

Definition 1. Let $P$ be a planar shape with a polygonal boundary, and let $\vec{a}=$ $(\cos \alpha, \sin \alpha)$ denotes the unit vector with direction $\alpha$. Then, the orientation of the shape is defined by the angle $\alpha$ such that the total sum

$$
F(\alpha, P)=\sum_{e \text { is an edge of } P}\left|\mathbf{p r}_{\vec{a}}(e)\right|^{2}
$$

of squared lengths of projections of all the edges of $P$ onto a line having the slope $\alpha$ is maximal possible.

Since the length of the projection $\mathbf{p r} \vec{a}\left(e_{i}\right)$ of the edge $e_{i}$ onto a line having the slope $\alpha$ is

$$
\left|\operatorname{pr}_{\vec{a}}\left(e_{i}\right)\right|=\left|e_{i}\right|\left|\left(\cos \alpha_{i} \cos \alpha+\sin \alpha_{i} \sin \alpha\right)\right|=\left|e_{i}\right|\left|\cos \left(\alpha_{i}-\alpha\right)\right|,
$$

the function $F(\alpha, P)$ that should be maximised (in order to compute the orientation of $P$ ) can be expressed as

$$
F(\alpha, P)=\sum_{i=1}^{n}\left|\mathbf{p r}_{\vec{a}}\left(e_{i}\right)\right|^{2}=\sum_{i=1}^{n}\left|e_{i}\right|^{2} \cos ^{2}\left(\alpha_{i}-\alpha\right) .
$$

By setting the first derivative $d F(\alpha, P) / d \alpha$ equal to zero it can be shown that both angles for which $F(\alpha, P)$ reaches its minimum and maximum satisfy

$$
\frac{\sin (2 \alpha)}{\cos (2 \alpha)}=\frac{\sum_{i=1}^{n}\left|e_{i}\right|^{2} \sin \left(2 \alpha_{i}\right)}{\sum_{i=1}^{n}\left|e_{i}\right|^{2} \cos \left(2 \alpha_{i}\right)} .
$$

Once again, for a detailed proof and more details we refer to 9 . 


\section{New Shape Elongation Measure for Polygonal Shapes}

Following the idea of the standard method for measuring shape elongation we define the new elongation measure as the ratio of the maximum and minimum value of the function that has been used for computing the shape orientation.

Definition 2. Let $P$ be a shape with a polygonal boundary. Then, the elongation of $P$ is defined as the ratio

$$
\mathcal{E}(P)=\frac{\max \{F(\alpha, P) \mid \alpha \in[0,2 \cdot \pi]\}}{\min \{F(\alpha, P) \mid \alpha \in[0,2 \cdot \pi]\}}
$$

of the maximum and minimum of the function $F(\alpha, P)$.

The new definition seems well motivated. For practical applications it would be a desirable property if $\mathcal{E}(P)$ is easily computable. We will show that the computation is straight forward, and more over it turns up that there is a closed formula for computing shape elongation as defined by (8).

Theorem 1. Let $P$ be a shape with a polygonal boundary. Then the new elongation measure of $P$ can be expressed as

$$
\mathcal{E}(P)=\frac{\sum_{1 \leq i \leq n}\left|e_{i}\right|^{2}+\sqrt{\left(\sum_{1 \leq i \leq n}\left|e_{i}\right|^{2} \cos \left(2 \alpha_{i}\right)\right)^{2}+\left(\sum_{1 \leq i \leq n}\left|e_{i}\right|^{2} \cdot \sin \left(2 \alpha_{i}\right)\right)^{2}}}{\sum_{1 \leq i \leq n}\left|e_{i}\right|^{2}-\sqrt{\left(\sum_{1 \leq i \leq n}\left|e_{i}\right|^{2} \cdot \cos \left(2 \alpha_{i}\right)\right)^{2}+\left(\sum_{1 \leq i \leq n}\left|e_{i}\right|^{2} \cdot \sin \left(2 \alpha_{i}\right)\right)^{2}}}
$$

where $e_{i}(1 \leq i \leq n)$ are edges of the boundary of $P$ and $\alpha_{i}(1 \leq i \leq n)$ are angles between the edges $e_{i}$ and the $x$-axis.

Proof. By using a simple trigonometric identity $\cos ^{2}(\alpha)=\frac{1+\cos 2 \alpha}{2}$ we can transform the optimising function $F(\alpha, P)$ from the form (6) into:

$$
\begin{aligned}
& F(\alpha, P)= \\
& \frac{1}{2} \cdot \sum_{1 \leq i \leq n}\left|e_{i}\right|^{2}+\frac{1}{2} \cdot \sum_{1 \leq i \leq n}\left|e_{i}\right|^{2}\left(\cos \left(2 \alpha_{i}\right) \cos (2 \alpha)+\sin \left(2 \alpha_{i}\right) \sin (2 \alpha)\right) .
\end{aligned}
$$

As already proved (see (7)), the angle values $\gamma$ for which $F(\alpha, P)$ reaches its minimum and maximum satisfy $\frac{\sin (2 \gamma)}{\cos (2 \gamma)}=\frac{\sum_{i=1}^{n}\left|e_{i}\right|^{2} \sin \left(2 \alpha_{i}\right)}{\sum_{i=1}^{n}\left|e_{i}\right|^{2} \cos \left(2 \alpha_{i}\right)}$. Now, using the trigonometric identities: $\sin (2 \varphi)=\frac{ \pm \tan (2 \varphi)}{\sqrt{1+\tan ^{2}(2 \varphi)}}$ and $\cos (2 \varphi)=\frac{ \pm 1}{\sqrt{1+\tan ^{2}(2 \varphi)}}$ 
we derive that $\cos (2 \gamma)$ and $\sin (2 \gamma)$ at the extreme points of $F(\alpha, P)$ can be expressed (together) as

$$
\begin{gathered}
\cos (2 \gamma)=\frac{ \pm \sum_{1 \leq i \leq n}\left|e_{i}\right|^{2} \cos \left(2 \alpha_{i}\right)}{\sqrt{\left(\sum_{1 \leq i \leq n}\left|e_{i}\right|^{2} \cos \left(2 \alpha_{i}\right)\right)^{2}+\left(\sum_{1 \leq i \leq n}\left|e_{i}\right|^{2} \sin \left(2 \alpha_{i}\right)\right)^{2}}} \\
\sin (2 \gamma)=\frac{ \pm \sum_{1 \leq i \leq n}\left|e_{i}\right|^{2} \sin \left(2 \alpha_{i}\right)}{\sqrt{\left(\sum_{1 \leq i \leq n}\left|e_{i}\right|^{2} \cos \left(2 \alpha_{i}\right)\right)^{2}+\left(\sum_{1 \leq i \leq n}\left|e_{i}\right|^{2} \sin \left(2 \alpha_{i}\right)\right)^{2}}} .
\end{gathered}
$$

Entering the last two equalities into (10) we derive that the minimum and maximum of $F(\alpha, P)$ can be expressed as

$$
\begin{aligned}
\frac{1}{2} \sum_{1 \leq i \leq n}\left|e_{i}\right|^{2}+\frac{1}{2} \sum_{1 \leq i \leq n}\left|e_{i}\right|^{2} \cdot \frac{ \pm \cos \left(2 \alpha_{i}\right) \cdot \sum_{1 \leq i \leq n}\left|e_{i}\right|^{2} \cos \left(2 \alpha_{i}\right)}{\sqrt{\left(\sum_{1 \leq i \leq n}\left|e_{i}\right|^{2} \cos \left(2 \alpha_{i}\right)\right)^{2}+\left(\sum_{1 \leq i \leq n}\left|e_{i}\right|^{2} \sin \left(2 \alpha_{i}\right)\right)^{2}}} \pm \sin \left(2 \alpha_{i}\right) \cdot \sum_{1 \leq i \leq n}\left|e_{i}\right|^{2} \sin \left(2 \alpha_{i}\right) \\
+\frac{1}{2} \sum_{1 \leq i \leq n}\left|e_{i}\right|^{2} \cdot \frac{\sqrt{\left(\sum_{1 \leq i \leq n}\left|e_{i}\right|^{2} \cos \left(2 \alpha_{i}\right)\right)^{2}+\left(\sum_{1 \leq i \leq n}\left|e_{i}\right|^{2} \sin \left(2 \alpha_{i}\right)\right)^{2}}}{\sqrt{\left(\sum_{1 \leq n}\right.}}
\end{aligned}
$$

or equivalently as

$$
\frac{1}{2} \cdot \sum_{1 \leq i \leq n}\left|e_{i}\right|^{2} \pm \frac{1}{2} \cdot \frac{\left(\sum_{1 \leq i \leq n}\left|e_{i}\right|^{2} \cos \left(2 \alpha_{i}\right)\right)^{2}+\left(\sum_{1 \leq i \leq n}\left|e_{i}\right|^{2} \sin \left(2 \alpha_{i}\right)\right)^{2}}{\sqrt{\left(\sum_{1 \leq i \leq n}\left|e_{i}\right|^{2} \cos \left(2 \alpha_{i}\right)\right)^{2}+\left(\sum_{1 \leq i \leq n}\left|e_{i}\right|^{2} \sin \left(2 \alpha_{i}\right)\right)^{2}}}
$$

Thus, we derived that the maximum and minimum of $F(\alpha, P)$ are as follows:

$$
\begin{aligned}
& \max \{F(\alpha, P) \mid \alpha \in[0,2 \pi]\}= \\
& \frac{1}{2} \cdot \sum_{1 \leq i \leq n}\left|e_{i}\right|^{2}+\frac{1}{2} \cdot \sqrt{\left(\sum_{1 \leq i \leq n}\left|e_{i}\right|^{2} \cdot \cos \left(2 \alpha_{i}\right)\right)^{2}+\left(\sum_{1 \leq i \leq n}\left|e_{i}\right|^{2} \cdot \sin \left(2 \alpha_{i}\right)\right)^{2}}
\end{aligned}
$$


and

$$
\begin{aligned}
& \min \{F(\alpha, P) \mid \alpha \in[0,2 \pi]\}= \\
& \frac{1}{2} \cdot \sum_{1 \leq i \leq n}\left|e_{i}\right|^{2}-\frac{1}{2} \cdot \sqrt{\left(\sum_{1 \leq i \leq n}\left|e_{i}\right|^{2} \cdot \cos \left(2 \alpha_{i}\right)\right)^{2}+\left(\sum_{1 \leq i \leq n}\left|e_{i}\right|^{2} \cdot \sin \left(2 \alpha_{i}\right)\right)^{2}} .
\end{aligned}
$$

This establishes the proof.

Lemma 1 considers two properties that encompass the new elongation measure. The proof is omitted because it follows directly from the definitions.

Lemma 1. The new elongation measure satisfies the following properties:

- $\mathcal{E}(P) \in[1, \infty)$ for each polygonal shape $P$;

- $\mathcal{E}(P)$ is invariant with respect to similarity transformations.

Remark. It is worth mentioning that the new elongation measure is valid for both open and closed polygons, as it considers the boundary of the polygonal shape. It can be applied to open polygonal lines, but also to the set of several polygonal lines. This enables the method to be applicable to shapes whose boundaries are not completely extracted. The reasons for an incomplete extracted boundary could be: the shape is partially overlaid, there are large similarities between background pixels and pixels belonging to the shape, etc.

\section{Experiments}

In the previous section we proposed a new shape elongation measure. It is naturally motivated and simple to compute. There is a closed formula (9) that expresses the elongation of a given polygonal shape as a function of the boundary edges and angles that those edges made with the $x$-axis. It performs well in some canonical cases. For example, let us consider a rectangle $T(a)$ having edge lengths $a$ and 1 . In accordance with (9) its measured elongation is

$$
\mathcal{E}(T(a))=\frac{1+a^{2}+\sqrt{\left(a^{2}-1\right)^{2}}}{1+a^{2}-\sqrt{\left(a^{2}-1\right)^{2}}}=\left\{\begin{array}{cll}
a^{2} & \text { if } & a>1 \\
1 & \text { if } & a=1 \\
1 / a^{2} & \text { if } & a<1
\end{array}\right.
$$

which is acceptable. In the limit cases where $a \rightarrow \infty$ and $a \rightarrow 0$ the rectangle degenerates into a line segment while the measured elongations tend to infinity. This behaviour is expected, and in fact preferred. In the case of $a=1$ the measured elongation is equal to 1 . In this case the rectangle degenerates into a square which is a 4-fold rotationally symmetric shape. Problems arising when working with manyfold rotationally symmetric shapes are discussed in 78 .

Next we give several shapes with their measured elongations. The new measure $\mathcal{E}$ is boundary based and it is more sensitive to noise or to boundary defects (e.g. 


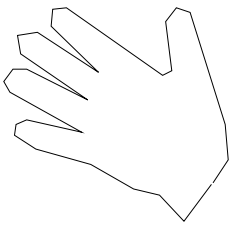

5.1173

(2.1218)

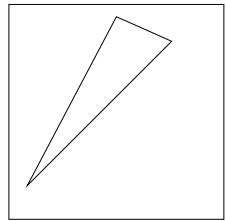

1.6553

(1.0604)

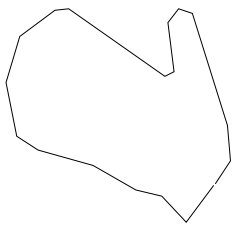

2.9167

(2.3975)

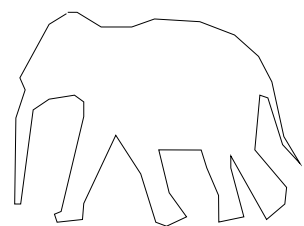

3.2768

(1.9653)

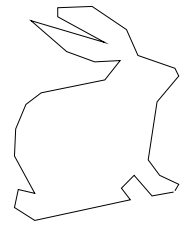

2.5274

(2.1093)

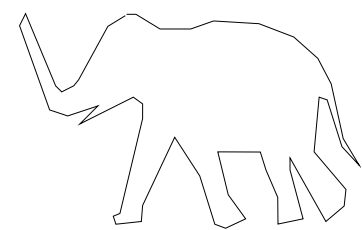

2.5775

(2.558)

Fig. 1. Computed elongations by the new method. Elongations computed by the standard method are in brackets.

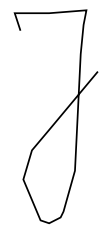

4.7473

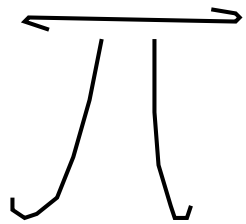

2.3787

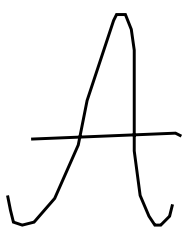

1.3609

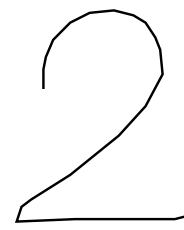

4.3489

Fig. 2. Computed elongations of polygonal lines by the new method

intrusions on the boundary) than the standard measure $\mathcal{E}_{s}$. That is illustrated by the first two examples from Fig.1. There is an essential difference between the measured elongations if the new measure $\mathcal{E}$ is used. On the other hand, there is only a small difference if those shapes are measured by the standard elongation measure $\mathcal{E}_{s}$. Such "sensitivity" is not necessarily a disadvantage - particularly when working in high precision (inspection) tasks.

The last two shapes in Fig.1 illustrate how shape deformations could affect the measured elongation. In those examples the rankings given by $\mathcal{E}$ and $\mathcal{E}_{s}$ are different. 
An advantage of the method is that it can be applied to shapes whose boundary consists of several polygonal lines (see the fourth shape in Fig.1.) or to shapes with missing parts on their boundaries (see the last example on Fig.2). The fourth shape in Fig.1 presents a square with a triangular hole. It has a measured elongation $\mathcal{E}_{s}$ very close to one. It is not surprising, because results from 78 imply that all $N$-fold rotationally symmetric shapes (if $N>2$ ) have the same, minimal possible, measured elongation which is equal to 1 . Since the percentage of pixels that correspond to the triangular hole is relatively small, it does not lead to an essential change in the measured elongation $\mathcal{E}_{s}$. If the new measure $\mathcal{E}$ is applied then the impact of the hole is more significant. That can be understood as a desirable property.

Several shapes that are presented usually by a curved line (or several of them) are given in Fig.2.

\section{Conclusion}

The traditional shape elongation measure is area based. It is therefore defined only for closed shapes. In this article, we proposed a shape boundary based measure, with a closed formula. Using our new method, elongation can be measured for any open shape, including shapes composed of several components. The measure is invariant with respect to rotation, translation and scaling.

\section{References}

1. Freeman, H., Shapira, R.: Determining the Minimum-Area Encasing Rectangle for an Arbitrary Closed Curve. Comm. of the ACM 18, 409-413 (1975)

2. Horn, B.K.P.: Robot Vision. MIT Press, Cambridge (1986)

3. Jain, R., Kasturi, R., Schunck, B.G.: Machine Vision. McGraw-Hill, New York (1995)

4. Klette, R., Rosenfeld, A.: Digital Geometry. Morgan Kaufmann, San Francisco (2004)

5. Martin, R.R., Stephenson, P.C.: Putting Objects into Boxes. Computer Aided Design 20, 506-514 (1988)

6. Rosin, P.L.: Techniques for Assessing Polygonal Approximations of Curves. IEEE Trans. PAMI 19(6), 659-666 (1997)

7. Tsai, W.H., Chou, S.L.: Detection of Generalized Principal Axes in Rotationally Symmetric Shapes. Pattern Recognition 24, 95-104 (1991)

8. Žunić, J., Kopanja, L., Fieldsend, J.E.: Notes on Shape Orientation where the Standard Method Does not Work. Pattern Recognition 39(5), 856-865 (2006)

9. Žunić, J.: Boundary Based Orientation of Polygonal Shapes. In: Chang, L.-W., Lie, W.-N. (eds.) PSIVT 2006. LNCS, vol. 4319, Springer, Heidelberg (2006) 